\title{
Mixed-Integer Vertex Covers on Bipartite Graphs
}

\author{
Michele Conforti ${ }^{1}$, Bert Gerards ${ }^{2,3}$, and Giacomo Zambelli ${ }^{1}$ \\ ${ }^{1}$ Dipartimento di Matematica Pura e Applicata, Universitá di Padova, Via Trieste \\ 63, 35121 Padova, Italy \\ conforti@math. unipd.it, giacomo@math.unipd.it \\ ${ }^{2}$ Centrum voor Wiskunde en Informatica, Kruislaan 413, 1098 SJ Amsterdam, \\ The Netherlands \\ Bert.Gerards@cwi.nl \\ 3 Technische Universiteit Eindhoven, Den Dolech 2, Eindhoven, The Netherlands
}

\begin{abstract}
Let $A$ be the edge-node incidence matrix of a bipartite graph $G=(U, V ; E), I$ be a subset of the nodes of $G$, and $b$ be a vector such that $2 b$ is integral. We consider the following mixed-integer set:

$$
X(G, b, I)=\left\{x: A x \geq b, x \geq 0, x_{i} \text { integer for all } i \in I\right\} .
$$

We characterize $\operatorname{conv}(X(G, b, I))$ in its original space. That is, we describe a matrix $(C, d)$ such that $\operatorname{conv}(X(G, b, I))=\{x: C x \geq d\}$. This is accomplished by computing the projection onto the space of the $x$ variables of an extended formulation, given in [1, for $\operatorname{conv}(X(G, b, I))$. We then give a polynomial-time algorithm for the separation problem for $\operatorname{conv}(X(G, b, I))$, thus showing that the problem of optimizing a linear function over the set $X(G, b, I)$ is solvable in polynomial time.
\end{abstract}

\section{Introduction}

Given a bipartite graph $G=(U, V ; E)$, a vector $b=\left(b_{e}\right)_{e \in E}$, with the property that $b$ is half-integral, i.e. $2 b_{e} \in \mathbb{Z}, e \in E$, and a set $I \subseteq(U \cup V)$, we consider the problem of characterizing the convex hull of all nonnegative $x \in \mathbb{R}^{U \cup V}$ such that

$$
\begin{aligned}
x_{i}+x_{j} & \geq b_{i j} \\
x_{i} & \in \mathbb{Z} \quad \text { for every } i j \in E,
\end{aligned}
$$

That is, given the edge-node incidence matrix $A$ of a bipartite graph $G$, a partition $(I, L)$ of its column-set, and an half-integral vector $b$, we consider the following mixed-integer set:

$$
X(G, b, I)=\left\{x: A x \geq b, x \geq 0, x_{i} \text { integer for all } i \in I\right\} .
$$

In this paper we provide a formulation for the polyhedron $\operatorname{conv}(X(G, b, I))$, where a formulation for a polyhedron $P$ is a description of $P$ as the intersection of a finite number of half-spaces. So it consists of a finite set of inequalities $C x \geq d$ such that $P=\{x: C x \geq d\}$. 
An extended formulation of $P$ is a formulation for a polyhedron $P^{\prime}$ in a higher dimensional space that includes the original space, so that $P$ is the projection of $P^{\prime}$ onto the original space.

A general technique to describe an extended formulation for the set of solutions of a system $A x \geq b$, when $A^{\top}$ is a network matrix and some of the variables are restricted to be integer, was introduced in 1 . In Section 2 we derive such an extended formulation for $\operatorname{conv}(X(G, b, I))$, while in Section 3 we describe a formulation in the original space by explicitly computing the projection of the polyhedron defined by the extended formulation. Finally, in Section 4 , we give a polynomial-time algorithm to solve the separation problem for $\operatorname{conv}(X(G, b, I))$.

\subsection{The Main Result}

Given a bipartite graph $G=(U, V ; E)$, a partition $(I, L)$ of $U \cup V$, and an halfintegral vector $b$, we say that a path $P$ of $G$ is an $I$-path if at least one endnode of $P$ is in $I$, and no intermediate node of $P$ is in $I$. We say that $P$ is odd if $P$ has an odd number of edges $e$ such that $b_{e}=\frac{1}{2} \bmod 1$. Whenever we have a vector $v$ with entries indexed by some set $S$, given a subset $T$ of $S$ we denote $v(T)=\sum_{i \in T} v_{i}$. In this paper we show the following:

Theorem 1. The polyhedron $\operatorname{conv}(X(G, b, I))$ is defined by the following inequalities:

$$
\begin{aligned}
x_{i}+x_{j} & \geq \quad b_{i j} \quad i j \in E, \\
2 x(V(P) \cap L)+x(V(P) \cap I) & \geq b(P)+\frac{1}{2} P \text { odd } I \text {-path }, \\
x_{i} & \geq 0 \quad i \in U \cup V .
\end{aligned}
$$

Eisenbrand 4 conjectured that the inequalities in (2)-(4) are sufficient to characterize $\operatorname{conv}(X(G, b, I))$ when $G$ is a path. Theorem 1 shows that this conjecture holds in a quite more general setting (and it certainly cannot be extended beyond that). Preliminary results for the path case were obtained by Skutella [1] and Eisenbrand [4].

\subsection{First Chvátal Closure}

The following observation allows us to describe $X(G, b, I)$ in terms of a pure integer set.

Observation 2. Let $\bar{x}$ be a vertex of $\operatorname{conv}(X(G, b, I))$. Then $2 \bar{x}$ is integral.

Proof: If not, let $U^{\prime}$ and $V^{\prime}$ be the sets of nodes $i$ in $U$ and $V$, respectively, such that $2 \bar{x}_{i}$ is not integer. Then, for $\epsilon$ small enough, the vectors $\bar{x}+\epsilon \chi^{U^{\prime}}-\epsilon \chi^{V^{\prime}}$ and $\bar{x}-\epsilon \chi^{U^{\prime}}+\epsilon \chi^{V^{\prime}}$ are both in $\operatorname{conv}(X(G, b, I))$, where we denote by $\chi^{S}$ the incidence vector of $S$ for any $S \subseteq U \cup V$.

Let $b^{\prime}=2 b, A^{\prime}$ be obtained form $A$ by multiplying by 2 the columns corresponding to nodes in $I$. By Observation 2, the linear transformation $x_{i}^{\prime}=x_{i}, i \in I$, 
$x_{i}^{\prime}=2 x_{i}, i \in L$, maps $X(G, b, I)$ into $\left\{x^{\prime}: A^{\prime} x^{\prime} \geq b^{\prime}, x^{\prime} \geq 0, x^{\prime}\right.$ integral $\}$, which is a pure integer set.

Let $P=v_{1}, \ldots v_{n}$ be an $I$-path. Notice that $b(P)=\frac{1}{2} \bmod 1$ is equivalent to $b^{\prime}(P)$ odd. Then the inequality

$$
\sum_{i \in V(P)} x_{i}^{\prime} \geq\left\lceil\frac{b^{\prime}(P)}{2}\right\rceil
$$

is a Gomory-Chvátal inequality of $\left\{x^{\prime}: A^{\prime} x^{\prime} \geq b^{\prime}, x^{\prime} \geq 0\right\}$. Indeed, assume $v_{1} \in I$. If $v_{n} \in I$, then (5) is obtained from

$$
\frac{1}{2}\left(2 x_{v_{1}}^{\prime}+x_{v_{2}}^{\prime} \geq b_{v_{1} v_{2}}^{\prime}\right)+\sum_{i=2}^{n-2} \frac{1}{2}\left(x_{v_{i}}^{\prime}+x_{v_{i+1}}^{\prime} \geq b_{v_{i} v_{i+1}}^{\prime}\right)+\frac{1}{2}\left(x_{v_{n-1}}^{\prime}+2 x_{v_{n}}^{\prime} \geq b_{v_{n-1} v_{n}}^{\prime}\right)
$$

by rounding up the right-hand-side. If $x_{n} \notin I$, then (5) is obtained from

$$
\frac{1}{2}\left(2 x_{v_{1}}^{\prime}+x_{v_{2}}^{\prime} \geq b_{v_{1} v_{2}}^{\prime}\right)+\sum_{i=2}^{n-1} \frac{1}{2}\left(x_{v_{i}}^{\prime}+x_{v_{i+1}}^{\prime} \geq b_{v_{i} v_{i+1}}^{\prime}\right)+\frac{1}{2}\left(x_{v_{n}}^{\prime} \geq 0\right)
$$

by rounding up the right-hand-side.

Furthermore the inequalities in (5) correspond to the inequalities in (3). Therefore Theorem 1 implies that the polyhedron defined by $A^{\prime} x^{\prime} \geq b^{\prime}, x^{\prime} \geq 0$ has Chvátal rank 1 . In the case where $G$ is a path with no intermediate node in $I$, this last fact follows immediately from a theorem of Edmonds and Jonhnson [23], since in this case $A^{\prime}$ satisfies the condition that the sum of the absolute values of the entries of each column is at most 2 .

\subsection{The Motivation}

A (general) mixed-integer set is a set of the form

$$
\left\{x \mid A x \geq b, x_{i} \text { integer } i \in I\right\}
$$

where $I$ is a subset of the columns of $A$ and $b$ is a vector that may contain fractional components.

In [1, it is shown that the problem of deciding if the above set is nonempty is NP-complete, even if $b$ is an half-integral vector and $A$ is a network matrix. (We refer the reader to [7] or [10 for definitions and results related to network matrices and, more generally, totally unimodular matrices.)

However, it may be possible that, when $A$ is the transpose of a network matrix, the associated mixed-integer programming problem is polynomially solvable. Indeed, let $M I X^{2 T U}$ be a mixed-integer set of the form (6) when $A^{\top}$ is a network matrix.

An extended formulation of the polyhedron $\operatorname{conv}\left(M I X^{2 T U}\right)$ was described in 1. The extended formulation involves an additional variable for each possible fractional part taken by the variables at any vertex of $\operatorname{conv}\left(M I X^{2 T U}\right)$. If this 
number is polynomial in the size of $(A, b)$, then such a formulation is compact, i.e. of polynomial size in the size of $(A, b)$. Therefore the problem of optimizing a linear function over $M I X^{2 T U}$ can be efficiently solved in this case. However, it seems to be rather difficult to compute the projection in the original $x$-space. It follows from Observation 2 that if $\bar{x}$ is a vertex of $\operatorname{conv}(X(G, b, I))$, then $\bar{x}_{i}-$ $\left\lfloor\bar{x}_{i}\right\rfloor \in\left\{0, \frac{1}{2}\right\}$. Therefore the extended formulation for $\operatorname{conv}(X(G, b, I))$ (which will be introduced in Section 2) is compact. The main contribution of this paper is the explicit description of the projection of the polyhedron defined by this extended formulation in the original $x$-space.

The mixed-integer set $X(G, b, I)$ is related to certain mixed-integer sets that arise in the context of production planning (see 9]). The case when $G$ is a star with center node in $L$ and leaves in $I$ has been studied by Pochet and Wolsey in [8, where they gave a compact extended formulation for the convex hull of feasible solutions. Günlük and Pochet [5] projected this formulation onto the original space, thus showing that the family of mixing inequalities gives the formulation in the $x$-space.

Miller and Wolsey [6] extended the results in [8] to general bipartite graphs, with the restriction that the partition $(I, L)$ coincides with the bipartition $(U, V)$ of the graph. Their result shows that the mixing inequalities associated with every single star of $G$ having center a node in $L$ and leaf nodes all nodes in $I$ give a formulation for this case.

\section{The Extended Formulation}

We use here a modeling technique introduced by Pochet and Wolsey [8] and extensively investigated in [1].

Observation 2 allows to express each variable $x_{i}, i \in L$, as

$$
x_{i}=\mu_{i}+\frac{1}{2} \delta_{i}, \mu_{i} \geq 0,0 \leq \delta_{i} \leq 1, \mu_{i}, \delta_{i} \text { integer. }
$$

For now, we assume $I=\emptyset$, that is, $L=(U \cup V)$.

Lemma 3. Let $i j \in E$, and suppose $x_{i}, x_{j}, \mu_{i}, \mu_{j}, \delta_{i}, \delta_{j}$ satisfy (7). If $b_{i j}=\frac{1}{2} \bmod 1, x_{i}, x_{j}$ satisfy $x_{i}+x_{j} \geq b_{i j}$ if and only if

$$
\begin{aligned}
\mu_{i}+\mu_{j} & \geq\left\lfloor b_{i j}\right\rfloor \\
\mu_{i}+\delta_{i}+\mu_{j}+\delta_{j} & \geq\left\lceil b_{i j}\right\rceil .
\end{aligned}
$$

If $b_{i j}=0 \bmod 1, x_{i}, x_{j}$ satisfy $x_{i}+x_{j} \geq b_{i j}$ if and only if

$$
\begin{aligned}
& \mu_{i}+\delta_{i}+\mu_{j} \geq b_{i j} \\
& \mu_{i}+\mu_{j}+\delta_{j} \geq b_{i j} .
\end{aligned}
$$

Proof: Assume $x_{i}, x_{j}, \mu_{i}, \mu_{j}, \delta_{i}, \delta_{j}$ satisfy (7). Then, if $b_{i j}=\frac{1}{2} \bmod 1$, constraint $x_{i}+x_{j} \geq b_{i j}$ is satisfied if and only if $\mu_{i}+\mu_{j} \geq\left\lfloor b_{i j}\right\rfloor$ and $\delta_{i}+\delta_{j} \geq 1$ whenever $\mu_{i}+\mu_{j}=\left\lfloor b_{i j}\right\rfloor$. If $b_{i j}=0 \bmod 1$, the constraint is satisfied if and only if $\mu_{i}+\mu_{j} \geq$ $b_{i j}-1$ and $\delta_{i}=\delta_{j}=1$ whenever $\mu_{i}+\mu_{j}=b_{i j}-1$.

It is easy to see that these conditions are enforced by the above constraints. 
Observation 4. Given $i j \in E$, the constraints (8) and (9) belong to the first Chvátal closure of the polyhedron defined by

$$
\begin{aligned}
\mu_{i}+\frac{1}{2} \delta_{i}+\mu_{j}+\frac{1}{2} \delta_{j} & \geq b_{i j} \\
\mu_{i}, \mu_{j} & \geq 0 \\
\delta_{i}, \delta_{j} & \leq 1 \\
\delta_{i}, \delta_{j} & \geq 0
\end{aligned}
$$

whenever $b_{i j}=\frac{1}{2} \bmod 1$ and $b_{i j}=0 \bmod 1$, respectively.

By applying the unimodular transformation $\mu_{i}^{0}=\mu_{i}, \mu_{i}^{1}=\mu_{i}+\delta_{i}$, the constraints $x_{i}=\mu_{i}+\frac{1}{2} \delta_{i}, \mu_{i} \geq 0,0 \leq \delta_{i} \leq 1$ become

$$
\begin{gathered}
x_{i}-\frac{1}{2}\left(\mu_{i}^{0}+\mu_{i}^{1}\right)=0 \\
\mu_{i}^{0} \geq 0 \\
0 \leq \mu_{i}^{1}-\mu_{i}^{0} \leq 1
\end{gathered}
$$

and constraints (8) and (9) become:

$$
\begin{gathered}
\mu_{i}^{0}+\mu_{j}^{0} \geq\left\lfloor b_{i j}\right\rfloor \\
\mu_{i}^{1}+\mu_{j}^{1} \geq\left\lceil b_{i j}\right\rceil \\
\mu_{i}^{1}+\mu_{j}^{0} \geq b_{i j} \\
\mu_{i}^{0}+\mu_{j}^{1} \geq b_{i j}
\end{gathered}
$$

Theorem 5. The projection onto the space of the $x$ variables of the polyhedron $Q$ defined on the space of the variables $\left(x, \mu^{0}, \mu^{1}\right)$ by the inequalities

$$
\begin{aligned}
& \text { (10), (11) for every } i \in U \cup V \text {, } \\
& (12) \text { for every } i j \in E \text { s.t. } b_{i j}=\frac{1}{2} \bmod 1 \\
& \text { (13) for every } i j \in E \text { s.t. } b_{i j}=0 \bmod 1
\end{aligned}
$$

is the polyhedron $\operatorname{conv}(X(G, b, \emptyset))$.

Proof: Since the variable $x_{i}$ is determined by (10) for all $i \in U \cup V$, we only need to show that the polyhedron defined by inequalities (11) for every $i \in U \cup V$, (12) for every $i j \in E$ s.t. $b_{i j}=\frac{1}{2} \bmod 1$, and (13) for every $i j \in E$ s.t. $b_{i j}=0 \bmod 1$, is integral. Let $A_{\mu}$ be the constraint matrix of the above system. Since $G$ is a bipartite graph, then the matrix $\bar{A}$, obtained by multiplying by -1 the columns of $A_{\mu}$ relative to the variables $\mu_{i}^{0}, \mu_{i}^{1}, i \in V$, has at most a 1 and at most a -1 in each row. Therefore $\bar{A}$ is the transpose of a network matrix, so $A_{\mu}$ is totally unimodular (see [10]). Since the right-hand-sides of (11)-(13) are all integer, the statement follows from the theorem of Hoffman and Kruskal.

Observation 6. For any $i \in U \cup V, x_{i}$ is integer valued if and only if $\delta_{i}=0$. Therefore, for a given $I \subseteq(U \cup V)$, the polyhedron $\operatorname{conv}(X(G, b, I))$ is the projection onto the space of the $x$ variables of the face $Q_{I}$ of $Q$ defined by the equations $\mu_{1}^{1}-\mu_{i}^{0}=0, i \in I$ (which correspond to $\delta_{i}=0, i \in I$ ). 


\section{The Formulation in the Original Space}

In this section we prove Theorem 1 by projecting the polyhedron $Q_{I}$ onto the space of the $x$ variables.

Let $p_{i}=\frac{\mu_{i}^{0}-\mu_{i}^{1}}{2}$. The $\mu_{i}^{0}=x_{i}+p_{i}$ and $\mu_{i}^{1}=x_{i}-p_{i}$. The inequalities (10)-(13), defining $Q$, become:

$$
\begin{array}{rlrl}
p_{i}+p_{j} & \geq\left\lfloor b_{i j}\right\rfloor-x_{i}-x_{j}, & i j \in E \text { s.t. } b_{i j}=\frac{1}{2} \bmod 1, \\
-p_{i}-p_{j} & \geq\left\lceil b_{i j}\right\rceil-x_{i}-x_{j}, & i j \in E \text { s.t. } b_{i j}=\frac{1}{2} \bmod 1, \\
p_{i}-p_{j} & \geq b_{i j}-x_{i}-x_{j}, & i j \in E \text { s.t. } b_{i j}=0 \bmod 1, \\
-p_{i}+p_{j} & \geq b_{i j}-x_{i}-x_{j}, & i j \in E \text { s.t. } b_{i j}=0 \bmod 1, \\
p_{i} & \geq-\frac{1}{2}, & i \in U \cup V, & \\
-p_{i} & \geq 0, & i \in U \cup V, & \\
p_{i} & \geq-x_{i}, & i \in U \cup V .
\end{array}
$$

By Observation 6. $\operatorname{conv}(X(G, B, I))$ is the projection onto the $x$-space of the polyhedron defined by the above inequalities and by $p_{i}=0$ for every $i \in I$.

Associate multipliers to the above constraints as follows:

$$
\begin{aligned}
& \left(u_{i j}^{++}\right) \quad p_{i}+p_{j} \geq\left\lfloor b_{i j}\right\rfloor-x_{i}-x_{j} \\
& \left(u_{i j}^{-}\right)-p_{i}-p_{j} \geq\left\lceil b_{i j}\right\rceil-x_{i}-x_{j} \\
& \left(u_{i j}^{+-}\right) \quad p_{i}-p_{j} \geq b_{i j}-x_{i}-x_{j} \\
& \left(u_{i j}^{-+}\right)-p_{i}+p_{j} \geq b_{i j}-x_{i}-x_{j} \\
& \left(u_{i}^{\frac{1}{2}}\right) \quad p_{i} \geq-\frac{1}{2} \\
& \left(u_{i}^{0}\right) \quad-p_{i} \geq 0 \\
& \left(u_{i}^{x}\right) \quad p_{i} \geq-x_{i}
\end{aligned}
$$

Any valid inequality for $\operatorname{conv}(X(G, b, I))$ has the form $\alpha_{u} x \geq \beta_{u}$, where

$$
\begin{gathered}
\alpha_{u} x=\sum_{b_{i j}=\frac{1}{2} \bmod 1}\left(u_{i j}^{++}+u_{i j}^{--}\right)\left(x_{i}+x_{j}\right)+ \\
\sum_{b_{i j}=0 \bmod 1}\left(u_{i j}^{+-}+u_{i j}^{-+}\right)\left(x_{i}+x_{j}\right)+\sum_{i \in U \cup V} u_{i}^{x} x_{i} \\
\beta_{u}=\sum_{b_{i j}=\frac{1}{2} \bmod 1}\left(u_{i j}^{--}\left\lceil b_{i j}\right\rceil+u_{i j}^{++}\left\lfloor b_{i j}\right\rfloor\right)+ \\
\sum_{b_{i j}=0 \bmod 1}\left(u_{i j}^{+-}+u_{i j}^{-+}\right) b_{i j}-\sum_{i \in L} \frac{1}{2} u_{i}^{\frac{1}{2}}
\end{gathered}
$$

for some nonnegative vector $u=\left(u_{i j}^{++}, u_{i j}^{--}, u_{i j}^{+-}, u_{i j}^{-+}, u_{i}^{\frac{1}{2}}, u_{i}^{0}, u_{i}^{x}\right)$ such that $u P=0$, where $P$ is the column-submatrix of the above system (14) involving columns corresponding to variables $p_{i}, i \in L$ (see e.g. Theorem 4.10 in [7]). For instance the inequality $x_{i}+x_{j} \geq b_{i j}$, for $i j \in E$ with $b_{i j}=\frac{1}{2} \bmod 1$, is obtained by setting $u_{i j}^{++}=u_{i j}^{--}=\frac{1}{2}$, and all other entries of $u$ to be 0 . 
We are interested in characterizing the nonnegative vectors $u$ such that $u P=0$ and $\alpha_{u} x \geq \beta_{u}$ is facet-defining for $\operatorname{conv}(X(G, b, I))$, and such that the inequality $\alpha_{u} x \geq \beta_{u}$ is not of the form $x_{i}+x_{j} \geq b_{i j}$, for some $i j \in E$, or $x_{i} \geq 0$, for some $i \in U \cup V$. From now on we will assume, w.l.o.g., that the entires of $u$ are integer and relatively prime.

We define an auxiliary graph $\Gamma_{u}=(L \cup\{d\}, F)$, where $d$ is a dummy node not in $U \cup V$, and $F$ is defined as follows.

- For every edge $i j \in E$ such that $i, j \in L$, there are $u_{i j}^{++}+u_{i j}^{--}+u_{i j}^{+-}+u_{i j}^{-+}$ parallel edges between $i$ and $j$ in $F$, each edge corresponding to a multiplier among $u_{i j}^{++}, u_{i j}^{--}, u_{i j}^{+-}, u_{i j}^{-+}$.

- For each node $i \in L$, there are $u_{i}^{\frac{1}{2}}+u_{i}^{0}+u_{i}^{x}+\sum_{j \in I: i j \in E}\left(u_{i j}^{++}+u_{i j}^{--}+\right.$ $\left.u_{i j}^{+-}+u_{i j}^{-+}\right)$parallel edges between $d$ and $i$ in $F$, each edge corresponding to a multiplier among $u_{i}^{\frac{1}{2}}, u_{i}^{0}, u_{i}^{x}$, or $u_{i j}^{++}, u_{i j}^{--}, u_{i j}^{+-}, u_{i j}^{-+}$, for some $j \in I$.

We impose a bi-orientation $\omega$ on $\Gamma_{u}$, that is, to each edge $e \in F$, and each endnode $i$ of $e$ that belongs to $L$, we associate the value $\omega(e, i)=$ tail if $e$ corresponds to an inequality of (14) where $p_{i}$ has coefficient -1 , while we associate the value $\omega(e, i)=$ head if $e$ corresponds to an inequality of (14) where $p_{i}$ has coefficient +1 . The dummy node $d$ is neither a tail nor a head of any edge. Thus, each edge of $\Gamma_{u}$ can have one head and one tail, two heads, two tails, or, if $d$ is one of the two endnodes, only one head and no tail or only one tail and no head.

For each $i \in L$, we denote with $\delta_{\omega}^{i n}(i)$ the number of edges in $F$ of which $i$ is a head, and with $\delta_{w}^{\text {out }}(i)$ the number of edges in $F$ of which $i$ is a tail.

We say that $\Gamma_{u}$ is $\omega$-eulerian if $\delta_{\omega}^{i n}(i)=\delta_{\omega}^{o u t}(i)$ for every $i \in L$.

Observation 7. $\Gamma_{u}$ is $\omega$-eulerian if and only if $u P=0$.

We define a closed $\omega$-eulerian walk in $\Gamma_{u}$ as a closed-walk in $\Gamma_{u}$,

$$
v_{0}, e_{0}, v_{1}, e_{1}, \ldots, v_{k}, e_{k}, v_{k+1}
$$

where $v_{0}=v_{k+1}$, with the property that $\omega\left(e_{h-1}, v_{h}\right) \neq \omega\left(e_{h}, v_{h}\right)$ for every $h$ such that $v_{h}$ is in $L, h=0, \ldots, k, k+1$, where the indices are taken modulo $k$. That is, if $v_{h} \in L$, then $v_{h}$ is a head of $e_{h-1}$ if and only if $v_{h}$ is a tail of $e_{h}$.

Observation 8. $\Gamma_{u}$ is $\omega$-eulerian if and only if $\Gamma_{u}$ is the disjoint union of closed $\omega$-eulerian walks. In particular, every node in $L \cup\{d\}$ has even degree in $\Gamma_{u}$.

Observe that, if $v_{0}, e_{0}, \ldots, e_{k}, v_{k+1}$ is a closed $\omega$-eulerian walk in $\Gamma_{u}$, then both graphs $\Gamma^{\prime}, \Gamma^{\prime \prime}$ on $L \cup\{d\}$ with edge-sets $F^{\prime}=\left\{e_{1}, \ldots, e_{k}\right\}$ and $F^{\prime \prime}=F \backslash F^{\prime}$, respectively, are $\omega$-eulerian. Suppose $F^{\prime \prime} \neq \emptyset$. Then there are nonnegative integer vectors $u^{\prime}$ and $u^{\prime \prime}$, both different from zero, such that $u^{\prime} P=0, u^{\prime \prime} P=0, \Gamma^{\prime}=\Gamma_{u^{\prime}}$ and $\Gamma^{\prime \prime}=\Gamma_{u^{\prime \prime}}$, and $u=u^{\prime}+u^{\prime \prime}$. By the fact that $\Gamma^{\prime}$ and $\Gamma^{\prime \prime}$ are $\omega$-eulerian, and by the structure of the inequalities in (14), the vectors $\left(\alpha_{u^{\prime}}, \beta_{u^{\prime}}\right)$ and $\left(\alpha_{u^{\prime \prime}}, \beta_{u^{\prime \prime}}\right)$ are both non-zero. Furthermore $\alpha_{u}=\alpha_{u^{\prime}}+\alpha_{u^{\prime \prime}}$ and $\beta_{u}=\beta_{u^{\prime}}+\beta_{u^{\prime \prime}}$, contradicting the fact that $\alpha_{u} x \geq \beta_{u}$ is facet-defining and the entries of $u$ are relatively prime.

Hence we have shown the following. 
Observation 9. Every closed $\omega$-eulerian walk of $\Gamma_{u}$ traverses all the edges in $F$. In particular, there exists a closed $\omega$-eulerian walk $v_{0}, e_{0}, \ldots, e_{k}, v_{k+1}$ of $\Gamma_{u}$ such that $F=\left\{e_{h} \mid h=1, \ldots, k\right\}$.

Suppose $d$ has positive degree in $\Gamma$. Then we may assume, w.l.o.g., that $v_{0}=$ $v_{k+1}=d$. Suppose $d=v_{h}$ for some $h=1, \ldots, k$. Then $v_{0}, e_{0}, v_{1}, \ldots, e_{h-1} v_{h}$ is a closed $\omega$-eulerian walk, contradicting the previous observation. Hence we have the following.

Observation 10. Node $d$ has degree 0 or 2 in $\Gamma_{u}$.

Next we show the following.

Lemma 11. Every node in $L \cup\{d\}$ has degree 0 or 2 in $\Gamma_{u}$.

Proof: We have already shown $d$ has degree 0 or 2 in $\Gamma_{u}$. If $d$ has degree 2, we assume $d=v_{0}=v_{k+1}$, else $v_{0}$ is arbitrarily chosen. If there is a node in $L$ with degree at least 4 , then there exists distinct indices $s, t \in\{1, \ldots, k\}$ such that $v_{s}=v_{t}$. We choose $s$ and $t$ such that $t-s$ is positive and as small as possible. Therefore $C=v_{s}, e_{s}, \ldots, e_{t-1}, v_{t}$ is a cycle of $\Gamma_{u}$ containing only nodes in $L$. Since $G$ is a bipartite graph, $C$ has even length, hence the edges in $C$ can be partitioned into two matchings $M_{0}, M_{1}$ of cardinality $|C| / 2$. We will denote with $H H, T T, H T$ the sets of edges of $F$ with, respectively, two heads, two tails, one head and one tail.

If $v_{s}$ is the head of exactly one among $e_{s}$ and $e_{t-1}$, then $C$ is a closed $\omega$ eulerian walk, contradicting Observation 9. Hence $v_{s}$ is either a head of both $e_{s}$ and $e_{t-1}$ or a tail of both $e_{s}$ and $e_{t-1}$. This shows that $|C \cap T T|=|C \cap H H| \pm 1$. Therefore there is an odd number of edges $e$ in $C$ such that $b_{e}=\frac{1}{2} \bmod 1$. By symmetry, we may assume $\sum_{e \in M_{0}} b_{e} \geq \sum_{e \in M_{1}} b_{e}+\frac{1}{2}$. Then the inequality

$$
2 \sum_{i \in V(C)} x_{i} \geq \sum_{e \in C} b_{e}+\frac{1}{2}
$$

is valid for $\operatorname{conv}(X(G, b, I))$, since it is implied by the valid inequalities $x_{i}+x_{j} \geq$ $b_{i j}, i j \in M_{0}$, because

$2 \sum_{i \in V(C)} x_{i}=2 \sum_{i j \in M_{0}}\left(x_{i}+x_{j}\right) \geq 2 \sum_{i j \in M_{0}} b_{i j} \geq \sum_{e \in M_{0}} b_{e}+\sum_{e \in M_{1}} b_{e}+\frac{1}{2}=\sum_{e \in C} b_{e}+\frac{1}{2}$.

Case 1: Node $v_{s}$ is a tail of both $e_{s}$ and $e_{t-1}$.

Then $|C \cap T T|=|C \cap H H|+1$, hence

$$
\sum_{e \in C \cap T T}\left\lfloor b_{e}\right\rfloor+\sum_{e \in C \cap H H}\left\lceil b_{e}\right\rceil+\sum_{e \in C \cap H T} b_{e}=\sum_{e \in C} b_{e}+\frac{1}{2} .
$$

Let $u^{\prime}$ be the vector obtained from $u$ as follows

$$
\left\{\begin{array}{l}
u_{i j}^{\prime * *}=u_{i j}^{* *}-1 \text { for every } i j \in C \\
u_{v_{s}}^{\prime 0}=u_{v_{s}}^{0}+2
\end{array}\right.
$$


all other components of $u^{\prime}$ and $u$ being identical, where $u_{i j}^{* *}$ is the variable among $u_{i j}^{++}, u_{i j}^{--}, u_{i j}^{+-}, u_{i j}^{-+}$corresponding to edge $i j$ of $C$.

Then one can easily see that $\Gamma_{u^{\prime}}$ is the graph obtained from $\Gamma_{u}$ by removing the edges $e_{s}, \ldots, e_{t}$, and adding two parallel edges $v_{s} d$ both with tail in $v_{s}$, hence $\Gamma_{u^{\prime}}$ is $\omega$-eulerian and $u^{\prime} P=0$. By (18)

$$
\beta_{u^{\prime}}=\beta_{u}-\sum_{e \in C} b_{e}-\frac{1}{2}
$$

while by construction

$$
\alpha_{u} x=\alpha_{u^{\prime}} x+2 \sum_{i \in V(C)} x_{i}
$$

Thus $\alpha_{u} x \geq \beta_{u}$ can be obtained by taking the sum of $\alpha_{u^{\prime}} x \geq \beta_{u^{\prime}}$ and (17), contradicting the assumption that $\alpha_{u} x \geq \beta_{u}$ is facet-defining.

Case 2: Node $v_{s}$ is a head of both $e_{s}$ and $e_{t-1}$.

Then $|C \cap T T|=|C \cap H H|-1$, hence

$$
\sum_{e \in C \cap T T}\left\lfloor b_{e}\right\rfloor+\sum_{e \in C \cap H H}\left\lceil b_{e}\right\rceil+\sum_{e \in C \cap H T} b_{e}=\sum_{e \in C} b_{e}-\frac{1}{2} .
$$

Let $u^{\prime}$ be the vector obtained from $u$ as follows

$$
\left\{\begin{array}{l}
u_{i j}^{\prime * *}=u_{i j}^{* *}-1 \text { for every } i j \in C \\
u_{v_{s}}^{\prime \frac{1}{2}}=u_{v_{s}}^{\frac{1}{2}}+2
\end{array}\right.
$$

all other components of $u^{\prime}$ and $u$ being identical.

Then one can easily see that $\Gamma_{u^{\prime}}$ is the graph obtained from $\Gamma_{u}$ by removing the edges $e_{s}, \ldots, e_{t}$, and adding two parallel edges $v_{s} d$ both with head in $v_{s}$, hence $u^{\prime} P=0$. By (19)

$$
\beta_{u^{\prime}}=\beta_{u}-\sum_{e \in C} b_{e}+\frac{1}{2}-2 \frac{1}{2}
$$

while by construction

$$
\alpha_{u} x=\alpha_{u^{\prime}} x+2 \sum_{i \in V(C)} x_{i}
$$

Thus $\alpha_{u} x \geq \beta_{u}$ can be obtained by taking the sum of $\alpha_{u^{\prime}} x \geq \beta_{u^{\prime}}$ and (17), contradicting the assumption that $\alpha_{u} x \geq \beta_{u}$ is facet-defining.

We are now ready to give the proof of the main theorem.

Proof of Theorem 1]. We show that all facet-defining inequalities $\alpha_{u} x \geq \beta_{u}$, where $u$ is nonnegative, integral, and with entries that are relatively prime, that are not inequalities in (2) or (4), are of the form (3). 
First we show the following.

$$
\sum_{i j \in E} u_{i j}^{--}>\sum_{i j \in E} u_{i j}^{++}+\sum_{i \in U \cup V} u_{i}^{\frac{1}{2}}
$$

In fact, we can write the inequality

$$
\alpha_{u} x \geq \sum_{b_{i j}=\frac{1}{2} \bmod 1}\left(u_{i j}^{--}+u_{i j}^{++}\right) b_{i j}+\sum_{b_{i j}=0 \bmod 1}\left(u_{i j}^{+-}+u_{i j}^{-+}\right) b_{i j}
$$

as nonnegative combination of inequalities of the form (2) or (4), therefore we must have

$$
\beta_{u}>\sum_{b_{i j}=\frac{1}{2} \bmod 1}\left(u_{i j}^{--}+u_{i j}^{++}\right) b_{i j}+\sum_{b_{i j}=0 \bmod 1}\left(u_{i j}^{+-}+u_{i j}^{-+}\right) b_{i j} .
$$

Thus

$$
\begin{aligned}
0 & <\beta_{u}-\sum_{b_{i j}=\frac{1}{2} \bmod 1}\left(u_{i j}^{--}+u_{i j}^{++}\right) b_{i j}-\sum_{b_{i j}=0 \bmod 1}\left(u_{i j}^{+-}+u_{i j}^{-+}\right) b_{i j} \\
& =\frac{1}{2}\left(\sum_{i j \in E} u_{i j}^{--}-\sum_{i j \in E} u_{i j}^{++}-\sum_{i \in U \cup V} u_{i}^{\frac{1}{2}}\right)
\end{aligned}
$$

which proves (20).

By Lemma (11) and Observation (9), $\Gamma_{u}$ consists of an induced cycle $C$ and isolated nodes, where every node in $V(C) \cap L$ is a head of exactly one edge and a tail of exactly one edge.

If $d$ is an isolated node, then each edge $i j$ of $C$ corresponds to a variable of the form $u_{i j}^{* *}$, and since the total number of heads in $C$ equals the number of tails, then $\sum_{i j \in E} u_{i j}^{--}=\sum_{i j \in E} u_{i j}^{++}$and $\sum_{i \in U \cup V} u_{i}^{\frac{1}{2}}=0$, contradicting (20). Thus we may assume that $C=v_{0}, e_{0}, \ldots, e_{k}, v_{k+1}$ where $d=v_{0}=v_{k+1}$.

Claim: The following are the only possible cases, up to symmetry.

1. Edges $d v_{1}, d v_{k}$ of $\Gamma_{u}$ correspond to variables $u_{v_{1}}^{x}$ and $u_{v_{k}}^{x}$, respectively;

2. $d v_{1}$ corresponds to variable $u_{w v_{1}}^{--}$or $u_{w v_{1}}^{-+}$for some $w \in I$, and $d v_{k}$ corresponds to $u_{v_{k}}^{x}$

3. $d v_{1}$ corresponds to variables $u_{w v_{1}}^{--}$or $u_{w v_{1}}^{-+}$for some $w \in I$, and $d v_{k}$ corresponds to variable $u_{w^{\prime} v_{k}}^{--}$or $u_{w^{\prime} v_{k}}^{-+}$for some $w^{\prime} \in I$.

Proof of claim. If $v_{1}$ is a head of $e_{0}$ and $v_{k}$ is a head of $e_{k}$, then the number of edges among $e_{1}, \ldots, e_{k-1}$ with two tails is one plus the number of edges with two heads. Since the former correspond to variables of type $u_{i j}^{--}$for some $i j \in E$, and the latter correspond to to variables of type $u_{i j}^{++}$for some $i j \in E$, then by (20) $d v_{1}$ does not correspond to variable $u_{v_{1}}^{\frac{1}{2}}$ or to a variable $u_{w v_{1}}^{++}$for any $w \in I$, and $d v_{k}$ does not correspond to variable $u_{v_{k}}^{\frac{1}{2}}$ or to a variable $u_{w v_{k}}^{++}$for any $w \in I$, thus one of the above three cases holds. 
If $v_{1}$ is a tail of $e_{0}$ and $v_{k}$ is a head of $e_{k}$, then the number of edges among $e_{1}, \ldots, e_{k-1}$ with two tails is equal the number of edges with two heads. By (20), $d v_{1}$ corresponds to variable $u_{w v_{1}}^{-}$for some $w \in I$, and $d v_{k}$ corresponds to either $u_{v_{k}}^{x}$ or to a variable $u_{w^{\prime} v_{k}}^{-+}$for some $w^{\prime} \in I$, thus case 2 or 3 holds.

If $v_{1}$ is a tail of $e_{0}$ and $v_{k}$ is a tail of $e_{k}$, then the number of edges among $e_{1}, \ldots, e_{k-1}$ with two tails is equal one minus the number of edges with two heads. By (20), $d v_{1}$ corresponds to variable $u_{w v_{1}}^{--}$for some $w \in I$, and $d v_{k}$ corresponds to a variable $u_{w^{\prime} v_{k}}^{--}$for some $w^{\prime} \in I$, thus case 3 holds. This completes the proof of the claim.

Case 1: Edges $d v_{1}$, $d v_{k}$ of $\Gamma_{u}$ correspond to variables $u_{v_{1}}^{x}$ and $u_{v_{k}}^{x}$, respectively.

In this case the path $P=v_{1}, e_{1}, \ldots, e_{k-1}, v_{k}$ of $\Gamma_{u}$ is also a path of $G$ containing only nodes in $L$, and $P$ contains an odd number of edges $e$ such that $b_{e}=$ $\frac{1}{2} \bmod 1$. The inequality $\alpha_{u} x \geq \beta_{u}$ is then $2 x(V(P)) \geq b(P)+\frac{1}{2}$. The edges of $P$ can be partitioned into two matchings $M_{0}$ and $M_{1}$, thus we may assume, w.l.o.g., $\sum_{e \in M_{0}} b_{e} \geq \sum_{e \in M_{1}} b_{e}+\frac{1}{2}$. Thus $2 x(V(P)) \geq 2 \sum_{i j \in M_{0}}\left(x_{i}+x_{j}\right) \geq$ $2 \sum_{i j \in M_{0}} b_{i j} \geq \sum_{e \in M_{0}} b_{e}+\sum_{e \in M_{1}} b_{e}+\frac{1}{2}=b(P)+\frac{1}{2}$, hence $\alpha_{u} x \geq \beta_{u}$ is not facet-defining.

Case 2: $d v_{1}$ corresponds to variable $u_{w v_{1}}^{--}$or $u_{w v_{1}}^{-+}$for some $w \in I$, and $d v_{k}$ corresponds to $u_{v_{k}}^{x}$.

In this case, $P=w, v_{1}, e_{1}, \ldots, e_{k-1}, v_{k}$ is an odd $I$-path of $G$ between $w \in I$ and $v_{k} \in L$. The inequality $\alpha_{u} x \geq \beta_{u}$ is $2 x(V(P) \cap L)+x_{w} \geq b(P)+\frac{1}{2}$, which is one of the inequalities in (3).

Case 3: $d v_{1}$ corresponds to variables $u_{w v_{1}}^{--}$or $u_{w v_{1}}^{-+}$for some $w \in I$, and $d v_{k}$ corresponds to variable $u_{w^{\prime} v_{k}}^{--}$or $u_{w^{\prime} v_{k}}^{-+}$for some $w^{\prime} \in I$.

If $w \neq w^{\prime}$, then the path $P=w, v_{1}, e_{1}, \ldots, e_{k-1}, v_{k}, w^{\prime}$ is an odd $I$-path of $G$ between $w \in I$ and $w^{\prime} \in I$. The inequality $\alpha_{u} x \geq \beta_{u}$ is $2 x(V(P) \cap L)+x_{w}+x_{w^{\prime}} \geq$ $b(P)+\frac{1}{2}$, which is one of the inequalities in (3).

If $w=w^{\prime}$, then we must have $v_{1} \neq v_{k}$, since otherwise $v_{1}$ would be either the head or the tail of both edges of $\Gamma_{u}$ incident to $v_{1}$. Thus $C^{\prime}=w, v_{1}, \ldots, v_{k}, w$ is a cycle of $G$. Since $G$ is a bipartite graph, $C^{\prime}$ has even length, hence the edges in $C^{\prime}$ can be partitioned into two matchings $M_{0}, M_{1}$ of cardinality $\left|C^{\prime}\right| / 2$. Since $C^{\prime}$ contains an odd number of edges $e$ such that $b_{w}=\frac{1}{2} \bmod 1$, then we may assume, w.l.o.g., $\sum_{e \in M_{0}} b_{e} \geq \sum_{e \in M_{1}} b_{e}+\frac{1}{2}$. The inequality $\alpha_{u} x \geq \beta_{u}$ is $2 x\left(V\left(C^{\prime}\right)\right) \geq b\left(C^{\prime}\right)+\frac{1}{2}$. But $2 x\left(V\left(C^{\prime}\right)\right)=2 \sum_{i j \in M_{0}}\left(x_{i}+x_{j}\right) \geq 2 \sum_{i j \in M_{0}} b_{i j} \geq$ $\sum_{e \in M_{0}} b_{e}+\sum_{e \in M_{1}} b_{e}+\frac{1}{2}=b\left(C^{\prime}\right)+\frac{1}{2}$, hence $\alpha_{u} x \geq \beta_{u}$ is not facet-defining.

\section{Separation}

Theorem 5 and Observation 6 imply that the problem of minimizing a linear function over the set $X(G, b, I)$ is solvable in polynomial time, since it reduces to solving a linear programming problem over the set of feasible points for (10)-(13). 
In this section we give a combinatorial polynomial-time algorithm for the separation problem for the set $\operatorname{conv}(X(G, b, I))$, thus giving an alternative proof that the problem of optimizing a linear function over such polyhedron, and thus over $X(G, b, I)$, is polynomial.

Clearly, given a nonnegative vector $x^{*}$, we can check in polynomial-time whether $x^{*}$ satisfies (2) for every edge. Thus, by Theorem 11, we only need to describe a polynomial-time algorithm that, given a nonnegative vector $x^{*}$ satisfying (2), either returns an inequality of type (3) violated by $x^{*}$, or proves that none exists.

For every $i j \in E$, let $s_{i j}^{*}=x_{i}^{*}+x_{j}^{*}-b_{i j}$. Since $x^{*}$ satisfies (2), then $s_{e}^{*}$ is nonnegative for every $e \in E$. Let $P=v_{1}, \ldots v_{n}$ be an odd $I$-path.

Claim. The vector $x^{*}$ satisfies $2 x^{*}(V(P) \cap L)+x^{*}(V(P) \cap I) \geq b(P)+\frac{1}{2}$ if and only if $s^{*}(P)+x^{*}\left(\left\{v_{1}, v_{n}\right\} \cap L\right) \geq \frac{1}{2}$.

Indeed, assume $v_{1} \in I$. If $v_{n} \in I$ then

$$
\sum_{i=1}^{n-1} s_{v_{i} v_{i+1}}^{*}=\sum_{i=1}^{n-1}\left(x_{v_{i}}^{*}+x_{v_{i+1}}^{*}-b_{v_{i} v_{i+1}}\right)
$$

gives the equality $s^{*}(P)=2 x^{*}(V(P) \cap L)+x^{*}(V(P) \cap I)-b(P)$, hence $2 x^{*}(V(P) \cap$ $L)+x^{*}(V(P) \cap I) \geq b(P)+\frac{1}{2}$ if and only if $s^{*}(P) \geq \frac{1}{2}$.

If $v_{n} \notin I$, then

$$
\sum_{i=1}^{n-1} s_{v_{i} v_{i+1}}^{*}+x_{v_{n}}^{*}=\sum_{i=1}^{n-1}\left(x_{v_{i}}^{*}+x_{v_{i+1}}^{*}-b_{v_{i} v_{i+1}}\right)+x_{v_{n}}^{*}
$$

gives the equality $s^{*}(P)+x_{v_{n}}^{*}=2 x^{*}(V(P) \cap L)+x^{*}(V(P) \cap I)-b(P)$, hence $2 x^{*}(V(P) \cap L)+x^{*}(V(P) \cap I) \geq b(P)+\frac{1}{2}$ if and only if $s^{*}(P)+x_{v_{n}}^{*} \geq \frac{1}{2}$.

This completes the proof of the Claim.

Therefore, if we assign length $s_{e}^{*}$ to every $e \in E$, we need to give an algorithm that, for any two nodes $r, t$ such that $r \in I$, either determines that the shortest odd $I$-path between $r$ and $t$ (if any) has length at least $\frac{1}{2}-x^{*}(\{t\} \cap L)$, or returns an odd $I$-path $P$ for which $2 x^{*}(V(P) \cap L)+x^{*}(V(P) \cap I)<b(P)+\frac{1}{2}$.

Observe that any walk $W$ between $r$ and $t$ that contains an odd number of edges $e$ such that $b_{e}=\frac{1}{2} \bmod 1$ either contains a sub-path $P$ that is an odd $I$-path or it contains a cycle $C$ that contains an odd number of edges $e$ such that $b_{e}=\frac{1}{2} \bmod 1$. In the former case, either both endnodes of $P$ are in $I$, or $t$ is the only endnode of $P$ in $L$. Hence, if $s^{*}(W)<\frac{1}{2}-x^{*}(\{t\} \cap L)$, then also $s^{*}(P)<\frac{1}{2}-x^{*}(\{t\} \cap L)$, hence $2 x^{*}(V(P) \cap L)+x^{*}(V(P) \cap I)<b(P)+\frac{1}{2}$. In the second case, since $G$ is bipartite, the edges of $C$ can be partitioned into two matchings $M_{0}$ and $M_{1}$ such that $b\left(M_{0}\right) \geq b\left(M_{1}\right)+\frac{1}{2}$. Thus $s^{*}(C)=\sum_{i j \in C}\left(x_{i}^{*}+x_{j}^{*}-b_{i j}\right)=2 x^{*}(V(C))-$ $b(C) \geq 2\left(x^{*}(V(C))-b\left(M_{0}\right)\right)+\frac{1}{2}=2 \sum_{i j \in M_{0}}\left(x_{i}^{*}+x_{j}^{*}-b_{i j}\right)+\frac{1}{2} \geq \frac{1}{2}$, hence $s^{*}(W) \geq \frac{1}{2}$.

Thus we only need to find, for every pair $r, t \in U \cup V$ with $r \in I$, the shortest walk $W$ between $r$ and $t$, w.r.t. the distance $s^{*}$, among all such walks containing an odd number of edges $e$ such that $b_{e}=\frac{1}{2} \bmod 1$. If, for a given choice of $r, t$, 
$s(W)<\frac{1}{2}-x^{*}(\{t\} \cap L)$, then by the above argument we can find in polynomial time a sub-path $P$ of $W$ such that $P$ is an odd $I$-path and $2 x^{*}(V(P) \cap L)+x^{*}(V(P) \cap I)<$ $b(P)+\frac{1}{2}$, otherwise we can conclude that $x^{*} \in \operatorname{conv}(X(G, b, I))$.

To conclude, we only need to show a polynomial-time algorithm that, given an undirected graph $\Gamma$ with nonnegative lengths on the edges $\ell_{e}, e \in E(\Gamma)$, a subset $F \subseteq E(\Gamma)$, and a pair of nodes $r, t \in V(\Gamma)$, determines the walk $W$ of minimum length between $r$ and $t$ such that $|E(W) \cap F|$ is odd, or determines that no such walk exists. The latter problem can be solved in polynomial time. Since, as far as we know, this fact is folklore, we briefly describe an algorithm.

We construct a new graph $\Gamma^{\prime}$ as follows. For every node $v \in V(\Gamma)$, there is a pair of nodes $v, v^{\prime}$ in $V\left(\Gamma^{\prime}\right)$. For every edge $u v \in E(\Gamma), E\left(\Gamma^{\prime}\right)$ contains the edges $u v^{\prime}$ and $u^{\prime} v$ if $u v \in F$, and the edges $u v$ and $u^{\prime} v^{\prime}$ if $u v \notin F$, each with length $\ell_{u v}$. One can verify that a walk $W$ between $r$ and $t$ with an odd number of edges in $F$ exists in $\Gamma$ if and only if there exists a walk of the same length between $r$ and $t^{\prime}$ in $\Gamma^{\prime}$. Hence we only need to find a shortest path between $r$ and $t^{\prime}$ in $\Gamma^{\prime}$, if any exists, and output the corresponding walk in $\Gamma$.

\section{References}

1. M. Conforti, M. Di Summa, F. Eisenbrand, L.A. Wolsey, Network formulations of mixed-integer programs, In preparation, 2006.

2. J. Edmonds and E.L. Johnson, Matching: a well-solved class of integer linear programs, Combinatorial Structures and Their Applications (R.K. Guy, et al., eds.), Gordon and Breach, New York, 1970, 89-92.

3. J. Edmonds and E.L. Johnson, Matching, Euler tours and the Chinese postman, Mathematical Programming 5 (1973), 88-124.

4. F. Eisenbrand, Mixed Integer Programming over TU systems, Manuscript, 2006.

5. O. Günlük and Y. Pochet, Mixing mixed integer inequalities, Mathematical Programming 90 (2001), 429-457.

6. A. Miller and L.A. Wolsey, Tight formulations for some simple MIPs and convex objective IPs, Mathematical Programming B 98 (2003), 73-88.

7. G.L. Nemhauser, L.A. Wolsey, Integer and Combinatorial Optimization, Wiley Interscience, New York, 1988.

8. Y. Pochet and L.A. Wolsey, Polyhedra for lot-sizing with Wagner-Whitin costs, Mathematical Programming 67 (1994), 297-324.

9. Y. Pochet and L.A. Wolsey, Production Planning by Mixed Integer Programming, Springer Series in Operations Research and Financial Engineering, New York, 2006.

10. A. Schrijver, Theory of Linear and Integer Programming, Wiley, New York, 1986.

11. M. Skutella, Mixed Integer vertex cover on paths, Manuscript, 2005. 